\title{
Perforation of aluminium foam core sandwich panels under impact loading: A numerical and analytical study
}

\author{
Ibrahim Elnasri ${ }^{1,2, a}$ and Han Zhao ${ }^{3}$ \\ ${ }^{1}$ Laboratoire de Génie Mécanique, Ecole Nationale d’Ingénieurs de Monastir, Av. Ibn ElJazzar, Monastir 5019, Tunisia \\ ${ }^{2}$ Institut Supérieur des Systèmes Industriels de Gabès, 214 rue Slaheddine Ayoubi, 6011 Gabès, Tunisia \\ ${ }^{3}$ Laboratoire de Mécanique et Technologie, ENS-Cachan/CNRS-UMR8535/Université Paris 6, 61, avenue du président \\ Wilson, 94235 Cachan Cedex, France
}

\begin{abstract}
This paper reports the numerical results of the inversed perforation test instrumented with Split Hopkinson Pressure Bar SHPB with an instrumented pressure bar on the AlSi7Mg0.5 aluminium foam core sandwich panels with $0.8 \mathrm{~mm}$ thick 2024 T3 aluminium top and bottom skin. The numerical models are developed in order to understand the origin of the enhancement of the top skin loads found under impact loading (paper published by [1]). Numerical predicted piercing force vs displacement curves are compared with experimental measurements (tests at impact velocities at 27 and $44 \mathrm{~m} / \mathrm{s}$ ). The simulation catches all process of the perforation of the sandwich panels (top skin, foam core, and bottom skin). Within experimental scatter, there is a good agreement between numerical predictions and experimental measurements. Virtual tests with different impact velocities up $200 \mathrm{~m} / \mathrm{s}$ are presented and showed a significant enhancement of the piercing force under impact loading (top skin peak and foam core plateau loads). In order to understand the origin of these force enhancements, any difference of detailed local information between static and dynamic loading is studied and showed that a shock front effect is responsible for the enhancement piercing force. An analytical model using an improved RPPL shock model based a power law densification assumption is proposed to calculate the top skin piercing force. The improved RPPL shock model agrees with the FE results for small velocities and gives better prediction of the piercing force than the RPPL shock model for large velocities $(>100 \mathrm{~m} / \mathrm{s})$.
\end{abstract}

\section{Introduction}

Sandwich plates and shells are important elements in modern lightweight construction. The main advantage of the sandwich principle is the fact that structures with high bending stiffness and rather low specific weight are obtained. The impact behaviours (energy absorption, perforation limits) are the important design features for sandwich panels used in aeronautics structures. The core materials of such panels (honeycombs, foams, hollow sphere agglomerates, etc.) play often dominant roles in the whole energy absorption as well as their penetration behaviour. [2] Conducted experimental and finite element studies on aluminium foam sandwich panels subjected to both quasi-static and impact loadings. Four deformation modes were summarised as global bending, localised indentation, localised indentation with global bending, and localised indentation with bending along clamping edge. However, key features of force-displacement curves, detailed deformation in face-sheets and foam core were not highlighted. [3] Carried out experimental tests and numerical simulations of the bird strike on sandwich panels made from aluminium foam core with aluminium face sheets. There is no perforation observed from the tests. [4] investigated the impact response of sandwich panels with Alporas foam cores and fibre-reinforced thermoplastic, and fibre-metal laminate (FML) facesheets. The indentation resistance of these sandwich panels

\footnotetext{
${ }^{a}$ Corresponding author: ibrahim.nasri@issig.rnu.tn
}

was found to be rate sensitive over the full range of conditions examined. Zhao et al. [1] tested the perforation behaviour of AlSi7Mg0.5 aluminium foam core sandwich panels with $0.8 \mathrm{~mm}$ thick $2024 \mathrm{~T} 3$ aluminium top and bottom skin using a split Hopkinson pressure bar, and recorded the piercing force displacement history. Quasistatic, impact tests (around $20 \mathrm{~m} / \mathrm{s}$ and around $45 \mathrm{~m} / \mathrm{s}$ ) were performed. A significant enhancement of the top skin peak loads under impact loading was observed, although the skin sheet as well as foam cores are nearly rate insensitive. They suggested that a possible reason is the difference of compressive strain level of foam core reached before the perforation of top skin under static and impact loading because of different face-foam core interaction mechanism. Such localised foam core strength enhancement leads to the increase of the top skin peak loads. However, no detailed quantitative analysis has been presented in this work and there is no detailed local information due to experimental difficulties to better understand the original of the enhancement of the top skin peak loads observed under impact loading. Additionally, the explanation of the puzzling results have not convinced the scientists, meaning that they still need an in depth study.

For this purpose, a numerical model is built and it is calibrated by testing the results to be sure that the main features of the perforation of the aluminium foam core sandwich panels are well represented. Afterwards, numerical testing at various impact velocities is performed and the local information, which are not experimentally available, (stress, strain, etc.) are analysed in order to 


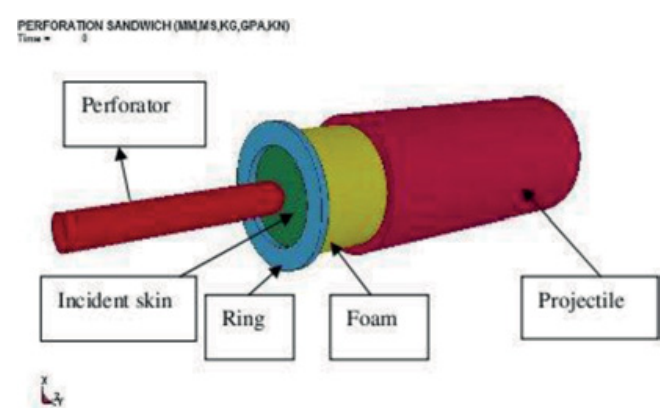

Figure 1. Numerical model for the perforation of the sandwich panels.

understand the piercing process. Finally, an analytical model using an improved RPPL shock model based on a power law densification assumption allows for an easy determination of its parameters from experimental data that are applied to calculate the resistance force during the perforation process.

\section{Numerical studies}

\subsection{Finite element modelling}

The numerical model is defined on the explicit code LS-DYNA [5]. Figure 1 shows the numerical model of the perforation of the sandwich panel which consists of 6 parts. The sandwich panel is integrated between two rigid parts. The aluminium hollow tube-like projectile (part 6) and aluminium clamping ring (part 2) are modelling as the rigid body by the material type $20 *$ (MAT_RIGID) in LS-DYNA codes. The rigid parts are merged for one Part 6. The perforator is represented as the elastic by the material type $1 *$ (MAT_ELASTIC, density $=8050 \mathrm{Kg} / \mathrm{m}^{3}$, Young moduli: $188 \mathrm{GPa}$, Poisson ratio $=0.3$ ). The aluminium 2024 T3 sheet are represented by shell elements with five integration points in the thickness. This model consists of 4800 2-D elements for the two sheets. The foam is modelled by using brick elements. This model consists of 90195 nodes, $76536-3 \mathrm{D}$ elements divided in 28416 for the part of the perforator, 120 for the two rigid parts and 48000 brick elements for the foam core. As the most important zone is the penetration area just under the perforator, the sandwich panels is modelled with a fine mesh along with the length. The number of the elements on the thick is 21 elements for the foam core. The number of the elements on the transverse direction is 51 elements for the sheet and for the foam core. Tiebreak constraints were applied between the faceplate and the foam core. Here, the nodal tiebreak failure in the interface was given by the following quadratic failure criterion;

$$
\left(\frac{\sigma_{t b}}{N_{t b}}\right)^{2}+\left(\frac{\tau_{t b}}{S_{t b}}\right)^{2} \geq 1
$$

where $\sigma_{t b}$ and $\tau_{t b}$ are the nodal normal and shear stress components and $N_{t b}$ and $S_{t b}$ are the strength of the adhesive in pure tension or pure shear, respectively. Here, $N_{t b}=S_{t b}=5 \mathrm{MPa}$ was used. Contact between SHPB perforator bar and sandwich plate was established using eroding surface to surface contact. To model

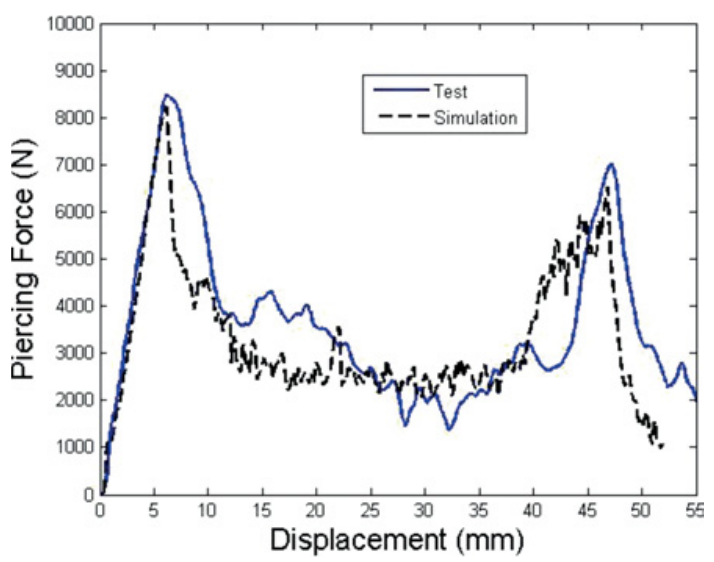

Figure 2. Comparison between experimental and simulated piercing force vs. displacement curves of the sandwich panel perforated at impact velocity $44 \mathrm{~m} / \mathrm{s}$.

contact between faceplate and the foam core after failure, eroding single surface contact was defined. Segment based contact option $(\mathrm{SOFT}=2)$ was used for simulating the sandwich plate impact. Frictionless contact was used in all simulations. All the parts in present work were modelled with single integration point (constant stress solid element, ElFORM = 1) because the reduced integration elements are robust choices for nonlinear analysis to overcome negative volumes, which are prone to happen in full integration elements. In spite of the afore mentioned benefits, the reduced integration elements suffer from non-physical spurious/hourglass modes. Control hourglass type 1 control cart is used to overcome this non-physical behaviour.

The boundary conditions in the impact simulation are the following: the perforator is fixed in $\mathrm{z}$-translation and z-rotation in degrees of freedom in the no-impact end. The contact between the sandwich panel and the two parts is defined as tied. The velocity of the sandwich panel and the projectile are modelled and put as initial velocity nodes for the sheets and for the foam and via the command INATIAL_RIGIG_BODY_VELOCITY for the projectile.

\subsection{Numerical models for the aluminum skin sheet and for cymat foam core}

The behaviour of the skin sheet is modelled by a damageable isotropic hardening elastic-plastic constitutive law proposed by [6], available in Ls-Dyna code [5] as the model of type 104 (MAT_DAMAGE_1). The parameters used for 2024 T3 aluminium sheet were identified from normalised tension tests by [7]. The foam core is modelled by a honeycomb constitutive model (MAT_26) available on the Ls-Dyna code with appropriate tensile failure strain. The normal stress vs. volumetric strain is obtained from quasi-static compression test (density $=235 \mathrm{~kg} / \mathrm{m}^{3}$, velocity $=0.01 \mathrm{~mm} / \mathrm{s}$ ). Due to the fact that they do not have a specific device to perform a shear test, the shear stress vs volumetric strain curve is deduced from the quasistatic compressive curve which is equal to the half. The fracture of the cell of the foam is achieved by using available tensile failure strain. Iteratively different values 

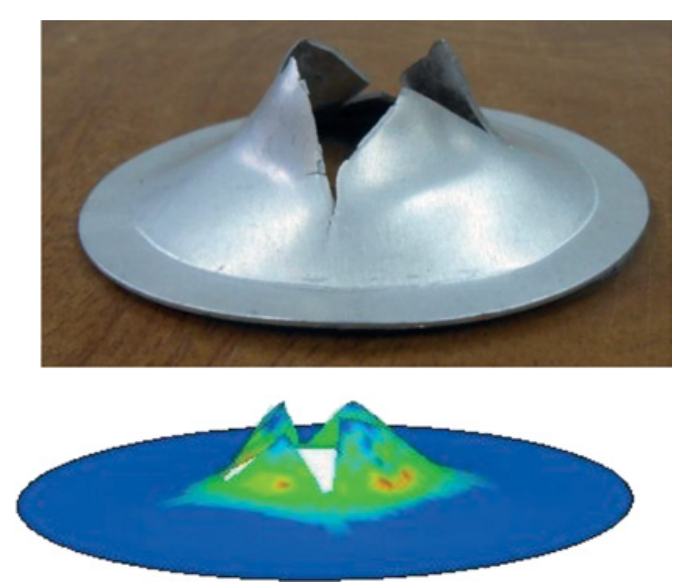

Figure 3. Breaking "petal" shape by simulation and by post mortem observation (Bottom skin, $\mathrm{v}=44 \mathrm{~m} / \mathrm{s}$ ).

were used to achieve the appropriate failure strain value. The constitutive properties of the foam are:

Density $=235 \mathrm{~kg} / \mathrm{m}^{3}$, Young Modulus $=70 \mathrm{GPa}$, Poisson ratio $=0.285$, SIGY $=240 \mathrm{MPa}, \quad \mathrm{Vf}=0.23, \mathrm{TSEL}=$ $5 \%$.

\subsection{Results and discussions}

With appropriate selection of constitutive model of the foam core, Fig. 2 shows the simulated piercing force-displacement curves at $44 \mathrm{~m} / \mathrm{s}$ compared with the experimental result. The piercing force is obtained by using the so-called command: *DATABSE_ NODAL FORCE_ GROUP. All nodes on the perforated end were integrated in the group nodes which can be compared to the piercing force recorded by the strain gauge instrumented on the Split Hopkinson Pressure Bar. It was observed that the simulated piercing curve was in good agreement with experiments. One can note that the simulated peak piercing force for the incident sheet is small lower than the experiment case but the difference was not significant to affect the final result and is apriority due to errors for the modelling. It can be seen the simulation catch ell the petalling failure mode observed on the experimental test (Fig. 3 for the bottom skin and Fig. 4 for the top skin).

\section{Analysis of virtual numerical tests}

The present numerical model provides the virtual tests where all the detailed information are not available in a real test (strain, stress, etc.) is known. Five numerical tests are conducted in dynamic loading up to the impact velocity $200 \mathrm{~m} / \mathrm{s}$. Piercing impact force vs. displacement curves for impact velocities $44,100,140$ and $180 \mathrm{~m} / \mathrm{s}$ are given in Fig. 5. It is observed that:

- The piercing top skin peak force and level of the plateau of the perforation of the foam core increases regularly with the impact speed. It indicates that this model contains probably the hints for the understanding of this force enhancement, which is probably due to the propagation of the shock front because the constitutive relation used for the foam cores as well as aluminium sheets are all rate insensitive.
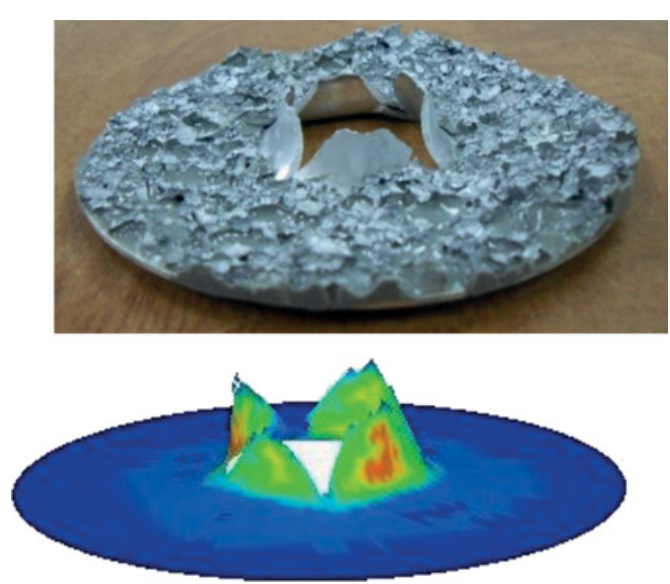

Figure 4. Breaking "petal" shape by simulation and by post mortem observation (top skin, $\mathrm{v}=44 \mathrm{~m} / \mathrm{s}$ ).

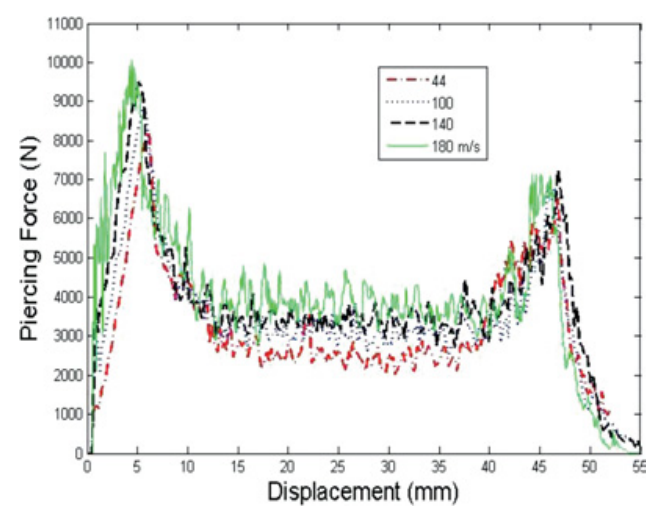

Figure 5. Comparison of simulated piercing force vs displacement curves of the sandwich panel perforated at 44, 100, 140 and $180 \mathrm{~m} / \mathrm{s}$.

- The maximum displacement of the top skin before breaking is unchanged with the impact velocity increase and the displacement of the bottom skin breaking is the same of all curves, these can prove that the foam core isn't sustained for a compression.

- The peak load of the piercing force of the bottom skin is nearly the same value for all curves; this is confirmed that the aluminium face sheet is rate insensitive.

In order to understand the origin of these force enhancements, any difference of detailed local Information between static and dynamic loading is interesting. A noticeable difference is to study the stress level in the of the foam core in the contact with the perforator. Figure 6 shows the equivalent Von Mises Stress vs displacement for one element located in the middle of the foam core. The coordinates of the element along the loading are $(0,2 \mathrm{~mm})$. A significant increase in stress amplitude is found for impact loading case and is in increase with impact velocity, the huge enhancement on the strength is prior due to the propagation of the shock front in the foam core, which is the possible reason, because the uncompressing of the foam core is confirmed by simulation, which leads to a higher local foam core strength under the perforator and can increase the top skin peak loads. 


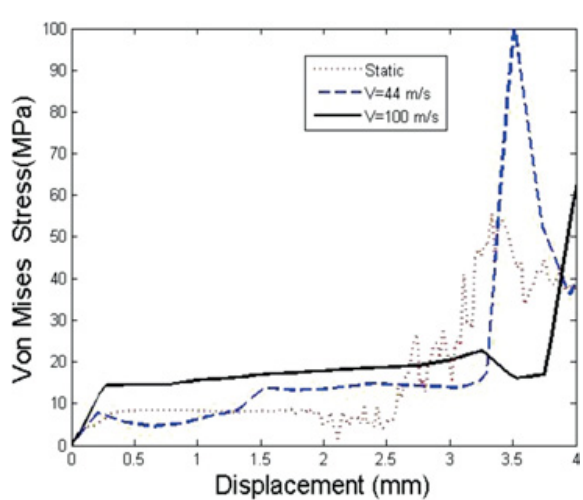

Figure 6. Comparison of Von Mises Stress vs. displacement curves for the element for three impact velocities (static, $\mathrm{v}=44 \mathrm{~m} / \mathrm{s}, \mathrm{v}=100 \mathrm{~m} / \mathrm{s}$ ). The coordinates of the element along the loading direction are $(0,2 \mathrm{~mm})$.

\section{Basic model of the impact piercing force enhancement}

In order to determine the model of the impact piercing force enhancement, analytical model for failure load predictions from [8] are considered for comparison with numerical predictions and experimental measurements. To take account the effect of the front shock, an improved shock model with a hardening locking proposed by [9] is also considered on the model. When the impact is normal to the surface of the sandwich panel, the motion of the projectile is:

$$
M \frac{d v}{d t}=-F_{p} .
$$

Where $F_{p}$ is the dynamic perforations force and $\mathrm{M}$ is is the mass of the projectile (sandwich panel+ hollow tubelike projectile+ ring). The dynamic perforation force can be expressed as the sum of the force needed to stretch the aluminium face sheet plate and the force needed to crush the foam beneath the indender $F_{\text {cruch }}$, the force required to tear the cells around the edge of the indenter $F_{\text {tear }}$ and the force enhancement caused by crushing the cellular material in the shock front $\mathrm{F}_{\text {shock}}$.

$$
F_{p}=F_{\text {strech }}+F_{\text {cruch }}+F_{\text {tear }}+F_{\text {shock }} .
$$

Where;

$$
F_{\text {strech }}=\sqrt{2} A l_{d f}\left(\varepsilon_{c r d f}\right)^{\frac{3}{2}} .
$$

Where:

$$
\begin{aligned}
& l_{d f}=\pi D_{C} . \\
& A=\frac{E h}{1-v^{2}} .
\end{aligned}
$$

Where $l_{d f}, \mathrm{~A}, \mathrm{E}, \mathrm{h}, v$ and $\varepsilon_{c r d f}$ are the length of the damage, stretching stiffness, Young's modulus, thickness, Poisson's ratio and dynamic fracture strain of the faceplate material, respectively, $D_{C}$ is the diameter of the volume crashed zone.

$$
F_{\text {cruch }}=\pi K D_{C}^{2} \sigma_{p l} / 4
$$

Where:

$\mathrm{K}$ the constraint factor for core crushing. [8] Use an

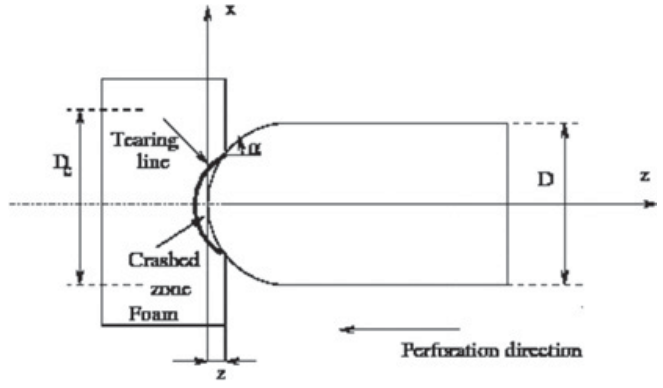

Figure 7. Cross-section of an axisymmetric perforator with a semi-spherical nose.

average value of 2.0 (from a range between 1.7 and 2.5) for the constraint factor, $\mathrm{K}$ in their work, which is based on the compression and indentation tests [10].

$\sigma_{p l}$ is the compression plateau stress.

$$
F_{\text {tear }}=\pi D_{C} \gamma
$$

Where:

$\gamma$ is the tearing energy per unit newly created area.

The tearing energy can be approximated by the following empirical formula:

$$
\gamma=550.8\left(\frac{\rho_{0}}{\rho_{s}}\right)^{1.8}\left(k J m^{-2}\right) .
$$

Where $\rho_{s}$ is the density of base material of aluminium Cymat foam. For aluminium Cymat foam with a nominal relative density of $8.7 \%$, the tear energy is $6.8 \mathrm{KJ} / \mathrm{m}^{2}$.

For the contribution of the force due to propagation of the front shock:

$$
F_{\text {shock }}=\frac{\pi D_{C}^{2} \rho_{0} v^{2}}{4 \varepsilon_{\text {shock }}} .
$$

Where:

$\rho_{0}$ is the density of the aluminium foam before crushing, $\varepsilon_{\text {shock }}$ is the shock strain, $\mathrm{v}$ is the impact velocity of the sandwich panel projectile.

$\mathrm{D}_{C}$ can be expressed by the following formula [10]:

$$
D_{C}=D+2[1.39 z \tan \alpha] .
$$

Where $\mathrm{D}$ is the diameter of the perforator, $\mathrm{z}$ is the indentation depth and $\alpha$ is the angle between the tearing line and the vertical axis. Here, we can use the value of the angle used in the indentation of the foam with semi-spherical nose $\left(\alpha=10.31^{\circ}\right)$. Figure 6 showed the simplified scheme of the perforation processes and the parameters presented in Eq. (11).

Substituting Eqs. (4)-(11) into Eq. (3) leads to:

$$
\begin{aligned}
F_{p}= & \pi D_{C}^{2}\left(\frac{4 \sqrt{2}}{D_{C}} A\left(\varepsilon_{c r d f}\right)^{\frac{3}{2}}+K \sigma_{p l}\right. \\
& \left.+\frac{4 \gamma}{D_{C}}+\frac{\rho_{0} v^{2}}{\varepsilon_{\text {shock }}}\right) / 4 .
\end{aligned}
$$

As it is mentioned by [9], the $\varepsilon_{\text {shock }}$ can be expressed en function of the basic continuity equation of the shock front:

$$
\sigma_{\text {shock }}-\sigma_{y}=\frac{\rho_{0} V_{\text {impact }}^{2}}{\varepsilon_{\text {shock }}} .
$$


Table 1. Identified parameters for power law locking.

\begin{tabular}{|l|c|l|l|}
\hline Material & Plateau stress (MPa) & $\mathrm{m}$ & $\mathrm{K}(\mathrm{MPa})$ \\
\hline Cymat foam core & 4 & 8.99 & 48.3 \\
\hline
\end{tabular}

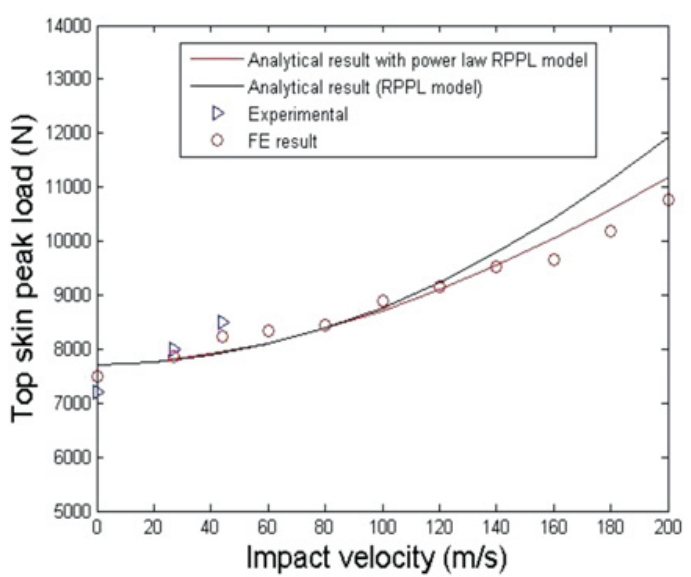

Figure 8. Comparison between FE results and the analytical models with power law RPPL model and with RPPL model the piercing force with the impact velocity with experimental results.

And the solution is defined with stress-strain of the materiel:

$$
\sigma_{\text {shock }}=f\left(\varepsilon_{\text {shock }}\right)
$$

And we can use a model using power law without locking strain to replace the RPPL model as used by [9]. The densification curve is defined by the initial yield stress $\sigma_{p l}$, the power $\mathrm{m}$, and the coefficient $\mathrm{K}$ :

$$
\sigma=\sigma_{p l}+k \varepsilon^{m} .
$$

Equations (13) and (14) then lead to:

$$
K \varepsilon_{\text {shock }}^{m}=\frac{\rho_{0} v^{2}}{\varepsilon_{\text {shock }}} .
$$

The derivation of the shock strain $\varepsilon_{\text {shock }}$ is straightforward:

$$
\varepsilon_{\text {shock }}=\left(\frac{\rho_{0} v^{2}}{k}\right)^{\frac{1}{m+1}} .
$$

From experimental stress-strain data, it is easy to identify such model. Table 1 provides parameters $(\mathrm{m}, \mathrm{k})$ for the studied Cymat foam. Substituting Eq. (17) into Eq. (12) leads to:

$$
\begin{aligned}
F_{p}= & \pi D_{C}^{2}\left(\left(4 \frac{\sqrt{2}}{D_{C}} A\left(\varepsilon_{c r d f}\right)^{\frac{3}{2}}+k \sigma_{p l}+\frac{4 \gamma}{D_{C}}+\ldots\right.\right. \\
& \left.+\left(\rho_{0} v^{2}\right)^{\frac{m}{m+1}} k^{\frac{1}{m+1}}\right) / 4
\end{aligned}
$$

The dynamic perforation force from Eq. (12) with RPPL and the modify shock theory model is compared with the corresponding results from FE model and the experimental results $(\mathrm{v}=27,44 \mathrm{~m} / \mathrm{s})$ as shown in Fig. 7 . The structure modifies shock theory and the RPPL models are agrees with FE results up impact velocities close to $100 \mathrm{~m} / \mathrm{s}$. It can observed that the RPPL overestimates the dynamic perforation load for large velocities $(<100 \mathrm{~m} / \mathrm{s})$ but the structure modify shock theory can ameliorate the prediction on the perforation force than the RPPL shock theory for a large impact velocities.

\section{Conclusions}

The numerical models of the inversed perforation test instrumented with SHPB with an instrumented pressure bar on the AlSi7Mg0.5 aluminum foam core sandwich panels with $0.8 \mathrm{~mm}$ thick $2024 \mathrm{~T} 3$ aluminum top and bottom skin are developed in order to understand the origin of the enhancement of the top skin loads found under impact loading (paper published by [1]).The numerical simulation is done using 3D finite element models in Ls-Dyna. The behaviour of the skin sheet is modeled by a damageable isotropic hardening elastic-plastic constitutive law as the model of type 104 (MAT_DAMAGE_1), where the foam core is modeled by a honeycomb constitutive model (MAT_26) with appropriate tensile failure strain.

Numerical predicted piercing force vs displacement curves are compared with experimental measurements (tests at impact velocities at 27 and $44 \mathrm{~m} / \mathrm{s}$ ). The simulation catch all processes of the perforation of the sandwich panels (top skin, foam, and bottom skin). Within experimental scatter, there is a good agreement between numerical predictions and experimental measurements. Virtual tests with different impact velocities up $200 \mathrm{~m} / \mathrm{s}$ are presented and showed:

- The piercing top skin peak force and level of the plateau of the perforation of the foam core increases regularly with the impact speed. It indicates that this model contains probably the hints for the understanding of this force enhancement, which is probably due to component interaction because the constitutive relation used for the foam cores as well as aluminum sheets are all rate insensitive.

- The maximum displacement of the top skin before breaking is unchanged with the impact velocity increase and the displacement of the bottom skin at breaking is the same of all simulated tests, these can prove that the foam core isn't sustained to a compression.

- The peak load of the piercing force of the bottom skin is nearly the same value for all curves; this is confirmed that the aluminum face sheet is rate insensitive.

In order to understand the origin of these force enhancements, a stress level in the element of the foam core on the contact with the perforator for three impact velocities (static, $40 \mathrm{~m} / \mathrm{s}$ and $100 \mathrm{~m} / \mathrm{s}$ ) for three impact velocities (static, $40 \mathrm{~m} / \mathrm{s}$ and $100 \mathrm{~m} / \mathrm{s}$ ) is analysed. A significant increase in the stress amplitude is found for impact loading case and is in increase with impact velocity, the huge enhancement on the strength is prior due to propagation of shock front in the foam core and is the one possible reason, because the uncompressing of the 
foam core is confirmed by simulation, which leads to a higher local foam core strength under the perforator and can increase the top skin peak loads and the foam core loads.

An analytical model using an improved RPPL shock model based on a power law densification assumption is proposed to calculate the top skin piercing and foam core piercing forces. The improved RPPL shock model agrees with the FE results for small velocities and gives better prediction of the piercing force than the RPPL shock model for large velocities ( $>100 \mathrm{~m} / \mathrm{s}$ ).

\section{References}

[1] H. Zhao, I. Elnasri, Y. Girard, I. J. of Impact Eng, 34 (7): 1246-57 (2007)

[2] W. Hou, H. Zhang, G. Lu, X. Huang X. In: Proceedings of the 6thAsia-Pacific conference on shock and impact load on structures, Perth, 7-9 December, p. 275-82 (2005)
[3] A.G. Hanssen, Y. Girard, L. Olovsson, T. Berstad, M. Langseth M., I. J. of Impact Eng. 32(7):1127-47 (2006)

[4] W.J. Cantwell, H. Kiratisaeve. J., Reinf Plast Comp; 24(10):1057-72 (2005)

[5] LS-DYNA keyword user's manual. Version 971(2007)

[6] Lemaitre, J. 2nd ed. Berlin: Springer, ISBN 3-54060980 (2006)

[7] S. Pattofatto, H.B, Zeng, H. Zhao. J. of Sand. Struct and Materiels (2011)

[8] M.S. Fat, K.S Park, Comp Struct; 52(3-4):353-64 (2001)

[9] S. Pattofatto, I. Elnasri, H. Zhao, H.Tsitsiris, F. Hild, Y. Girard, J. Mech. Phys. Solids, 55; 2672-2686 (2007)

[10] Reddy T.Y., Soden P.D., Reid S.R., Sadighi M. Collaborative research program on the costeffective use of fibre-reinforced composite offshore, marintech research, phase1, report no. CP04 (1991) 